\title{
Wheat Leaf Area Index Prediction Using Data Fusion Based on High-Resolution Unmanned Aerial Vehicle Imagery
}

\section{Shuang Wu}

Capital Normal University https://orcid.org/0000-0002-5996-4600

Lei Deng ( $\nabla$ denglei@cnu.edu.cn )

Capital Normal University https://orcid.org/0000-0002-4574-7381

Lijie Guo

Capital Normal University

\section{Yanjie Wu}

Capital Normal University

\section{Research}

Keywords: leaf area index (LAl), unmanned aerial vehicle (UAV), high resolution, data fusion

Posted Date: December 20th, 2021

DOI: https://doi.org/10.21203/rs.3.rs-1169494/v1

License: (a) (i) This work is licensed under a Creative Commons Attribution 4.0 International License. Read Full License 


\title{
Wheat Leaf Area Index Prediction using data fusion based on
}

\section{High-Resolution Unmanned Aerial Vehicle Imagery}

\author{
Shuang $\mathrm{Wu}^{1,2,3}$, Lei Deng ${ }^{1,2,3^{*}}$, Lijie Guo ${ }^{1,2,3}$, Yanjie $\mathrm{Wu}^{1,2,3}$ \\ *Correspondence: Lei Deng (denglei@cnu.edu.cn)
}

\section{Abstract}

Background: Leaf Area Index (LAI) is half of the amount of leaf area per unit horizontal ground surface area. Consequently, accurate vegetation extraction in remote sensing imagery is critical for LAI estimation. However, most studies do not fully exploit the advantages of Unmanned Aerial Vehicle (UAV) imagery with high spatial resolution, such as not removing the background (soil and shadow, etc.). Furthermore, the advancement of multi-sensor synchronous observation and integration technology allows for the simultaneous collection of canopy spectral, structural, and thermal data, making it possible for data fusion.

Methods: To investigate the potential of high-resolution UAV imagery combined with multi-sensor data fusion in LAI estimation. High-resolution UAV imagery was obtained with a multi-sensor integrated MicaSense Altum camera to extract the wheat canopy's spectral, structural, and thermal features. After removing the soil background, all features were fused, and LAI was estimated using Random Forest and Support Vector Machine Regression.

Result: The results show that: (1) the soil background reduced the accuracy of the LAI prediction, and soil background could be effectively removed by taking advantage of high-resolution UAV imagery. After removing the soil background, the LAI prediction accuracy improved significantly, $\mathrm{R}^{2}$ raised by about 0.27 , and RMSE fell by about 0.476 . (2) The fusion of multi-sensor synchronous observation data improved LAI prediction accuracy and achieved the best accuracy 
$\left(\mathrm{R}^{2}=0.815\right.$ and $\left.\mathrm{RMSE}=1.023\right)$. (3) When compared to other variables, CHM, NRCT, NDRE, and BLUE are crucial for LAI estimation. Even the simple Multiple Linear Regression model could achieve high prediction accuracy $\left(\mathrm{R}^{2}=0.679\right.$ and $\left.\mathrm{RMSE}=1.231\right)$, providing inspiration for rapid and efficient LAI prediction.

Conclusions: The method of this study can be transferred to other sites with more extensive areas or similar agriculture structures, which will facilitate agricultural production and management.

Keywords: leaf area index (LAI); unmanned aerial vehicle (UAV); high resolution; data fusion

\section{Background}

Wheat is the most widely grown grain crop in the world, and it plays an essential role in the food supply, accounting for approximately $20 \%$ of total energy consumption [1-3]. As the primary photosynthetic organ, the leaves of wheat have a significant impact on the overall growth. Leaf area index (LAI), as an essential parameter of wheat growth, can provide dynamic information during wheat growth. It is a critical metric for assessing crop growth and is closely related to the aboveground biomass and yield [4-7]. As a result, rapid, accurate, and non-destructive prediction of LAI is critical for field management. The traditional method of obtaining LAI is through artificial ground destructive sampling, which is time-consuming, labor-intensive, and hinders crop growth. The advancement of remote sensing technology in recent years has provided a new means for the rapid acquisition of LAI $[8,9]$.

Because LAI is half the amount of leaf area per unit horizontal ground surface area [10], accurate vegetation extraction in remote sensing imagery is critical. The vegetation information of crop canopy extracted from imagery is primarily determined by the combined effects of vegetation types, soil properties, shadows, and other factors [11]. The background (soil, weeds, and shadow) 
accounts for a certain proportion of the plot area in crop growth. The soil background exists throughout the crop growth cycle. Not only does the soil background account for a large proportion of the plot area in the early stages of crop growth, but exposed soil is also found in the late stages of crop growth due to differences in some factors (such as seedling emergence rate) between crops. Previously, some researchers attempted to estimate crop LAI using satellite remote sensing imagery, such as Landsat and Sentinel-2 satellite imagery [12-14]. Kamenova et al. [13], used various vegetation indices (VIs) extracted from Sentinel-2 multispectral imagery to establish the LAI prediction model, and LAI of winter wheat can be better estimated. Meyer et al [14]. found that the raw value of bands and VIs extracted from Landsat8-OLI multispectral imagery were used to establish a prediction model that can effectively predict the LAI of the temperate deciduous broad-leaved forest.

It can be seen that research on satellite remote sensing has made progress. However, satellite imagery was limited by spatial resolution. The extracted vegetation information is usually mixed with background information such as soil, resulting in the wrong calculation of leaf area per unit horizontal surface area. Thus, the estimated LAI value of the crop is inaccurate. In recent years, Unmanned Aerial Vehicle (UAV) remote sensing can obtain centimeter-level high spatial resolution imagery, better distinguishing vegetation, and background information, and is often used to estimate crop traits [15-17]. However, most UAV remote sensing studies currently still use the way of satellite remote sensing, which means that the extracted vegetation information without background processing is directly used in crop trait estimation, resulting in inaccurate LAI estimation $[18,19]$. Fortunately, some studies have noted the influence of the soil background. The remote sensing imagery of the UAV is processed in advance to remove the soil background. Still, 
this operation is only applied to estimating the crop's chlorophyll content [16] or yield [20], and the estimation of the crop's LAI is little. Furthermore, previous research has shown that it is difficult to observe a variety of data synchronously using UAV remote sensing. To address this issue, the typical solution is to carry multiple sensors (multispectral and thermal infrared sensors, etc.) on the same UAV platform and calibrate the generated multiple imageries using ground control points (GCPs), which is inefficient. For example, Manimatijiang et al. [20], the Mapir Survey2 RGB and FLIR Vue Pro r 640 cameras were installed on the DJI S100 + UAV platform to obtain visible and thermal infrared imagery, respectively. And then, GCPs were used to calibrate two kinds of images to obtain spectral and thermal information. The integration of tiny sensors on UAV is increasing rapidly, UAV can be equipped with multiple imaging sensors and GPS systems to obtain many data sets (RGB imagery, 3D points clouds and thermal imagery, etc.) simultaneously. It has become one of the most competitive tools, providing excellent possibilities for precision agriculture [21-23]. A typical example, such as the Micasense Altum camera, is the integration of multispectral and thermal infrared cameras into a single unit, which has the advantage of overlapping fields of view and simultaneous access to canopy spectral, structural, and thermal information [24]. Although a variety of data can be obtained by using the sensors integrated with UAV, few studies on their comprehensive utilization/fusion and potential in LAI application is still unknown.

Many regression methods based on statistics and machine learning, such as Partial Least Squares Regression (PLSR) [25], Artificial Neural Network (ANN) [26], and Random Forest Regression (RFR) [27], are currently used in crop trait estimation to realize the fusion of multi-sensor data [18]. However, most studies tend to add all the features extracted from remote 
sensing data to the model for training in data fusion using machine learning methods. The advantages of this are that it considers all the features, but it does not consider how adding them may affect the model's efficiency and lengthen its running time [28,29]. Previous studies have found different important features for different crops $[18,20]$. For example, Lee et al. [18] found that when using RFR to predict the nitrogen content of maize, the VIs such as Modified Simple Ratio (MSR), Wide Dynamic Range Vegetation Index (WDRI), and Ratio Vegetation Index (RVI) performed well. Maimaitijiang et al [20] discovered that canopy height and vegetation coverage extracted from UAV imagery performed well in estimating aboveground biomass and LAI of soybean. However, there have been few studies on the critical features of wheat LAI estimation [30]. Furthermore, most studies have shown that Multiple Linear Regression (MLR) is used to estimate crop traits rapidly. Because it has fast modeling speed, does not require very complex calculations, and still runs fast in the case of large amounts of data. However, there are few studies on the potential of MLR in the rapid estimation of wheat LAI, and few can propose a simple model for wheat LAI, that is, using a few important features with MLR.

The main purpose of this study is to find a method to estimate LAI with high precision by making full use of the advantages of UAV remote sensing, which means using the advantage of high spatial resolution of UAV imagery combined with multi-sensor synchronous observation. The specific objectives are as follows: (1) investigate the influence of soil background on LAI estimation and the role of high spatial resolution UAV imagery in removing soil background; (2) evaluate the potential of data fusion based on multi-sensor synchronous observation in LAI estimation and find an efficient and straightforward LAI estimation model based on the fused data. 
$111 \quad$ Materials

\section{Test Site and Filed Layout}

The study area is located in wheat-growing farmland in Xi county, Henan Province, China,

114 and covers an area of approximately $3565.497 \mathrm{~m}^{2}$, as shown in Figure 1 . The climate in the region

115 is subtropical monsoon, with an annual average temperature of $15.5{ }^{\circ} \mathrm{C}$, average yearly rainfall of more than $1200 \mathrm{~mm}$, and a daily average temperature of $10{ }^{\circ} \mathrm{C}$. As a result, it is ideal for wheat

117 planting and growth in this case. The wheat was planted in October and harvested the following

118 June. The experiment was conducted here on May 1, 2021. Currently, wheat was in the heading

119 stage of the growth period, with dark green leaves. To further facilitate establishing and verifying the LAI prediction model, $802 \mathrm{~m} \times 2 \mathrm{~m}$ plots were designed, and the ground data was measured on

12180 plots. At the same time, 8 GCPs are arranged in the study area for UAV imagery correction and registration, as shown in Figure 1. 


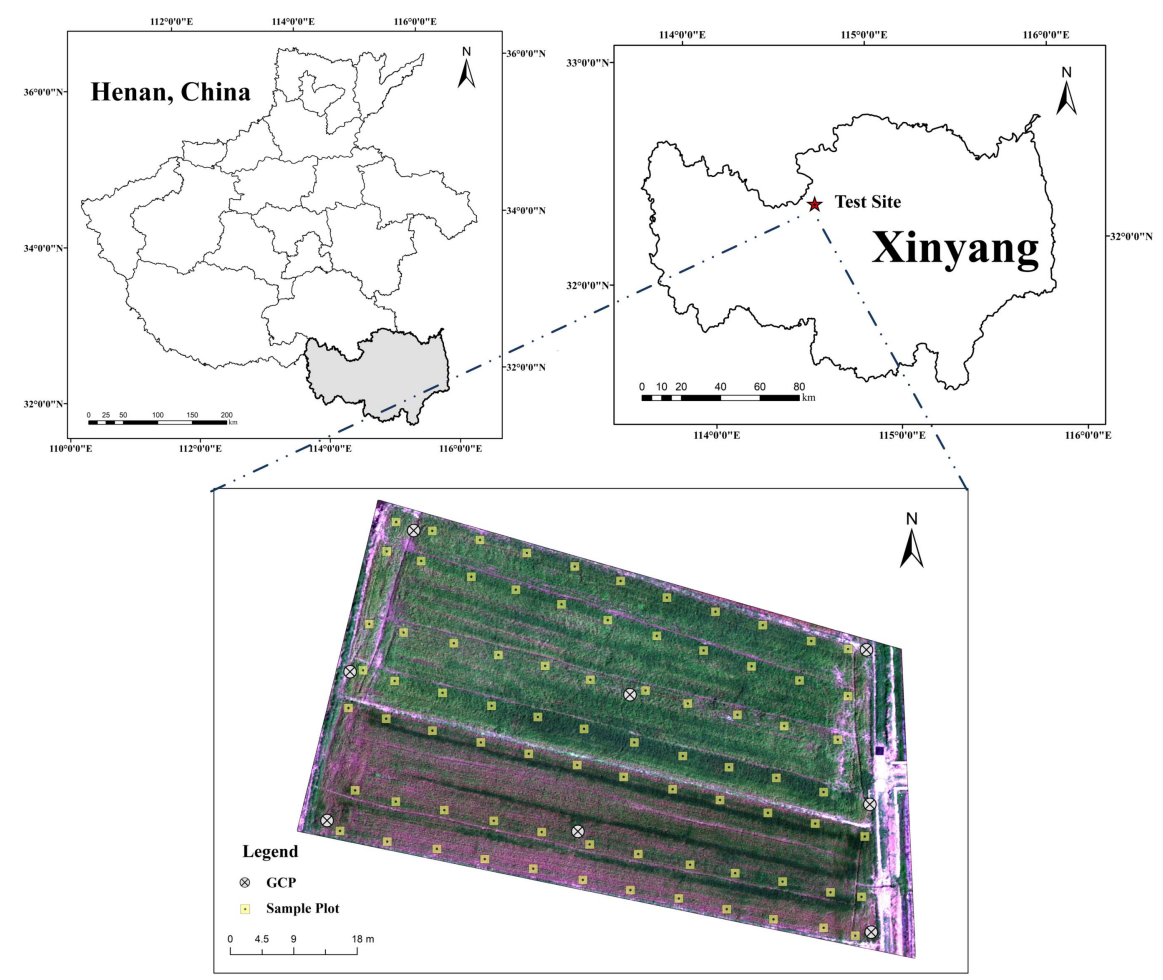

123

Fig. 1 Test site of the wheat fields shown using red, green, blue (RGB) sensor mosaic imagery taken on 1 May in Xinyang County, Henan Province, China.

\section{Field Data Acquisition}

LAI of 80 plots was measured by LAI-2200C Plant Crown Analyzer (LI-COR Inc., Lincoln, NE, USA) on May 1, 2021. The field data are obtained according to the measurement guidelines recommended in the instrument manual. 23 LAI values are randomly collected from each plot and then averaged to represent the LAI values of each plot. The number of plots within a certain LAI value range is counted, as shown in Figure 2. It is shown that the LAI of 80 plots is different. The LAI values are concentrated in the ranges of $1-2,2-3$, and $6-7$, with 20,13 , and 12 , respectively. The LAI value is in the range of 7-8, and the number of plots is two. 


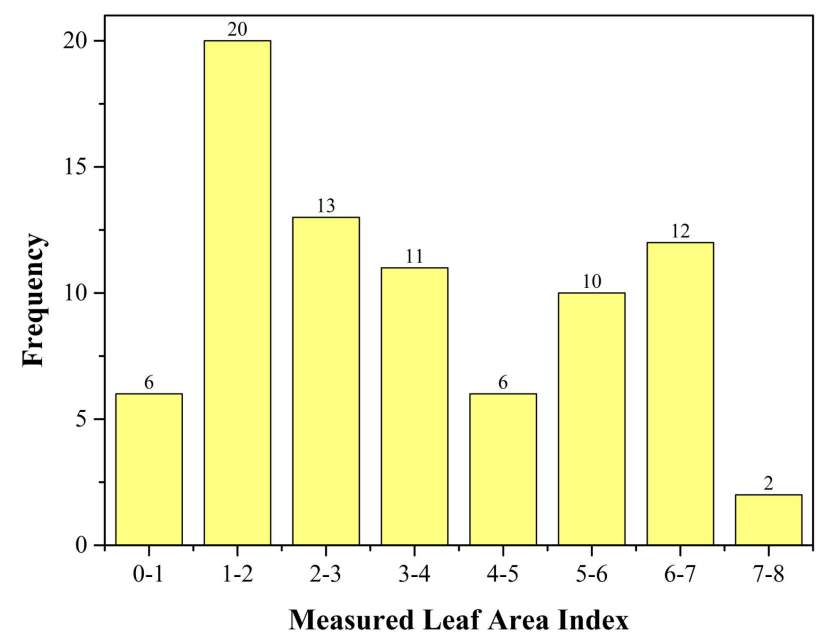

Fig. 2 Number of plots with different measured leaf area index area.

\section{UAV Imagery Acquisition and Processing}

The UAV imagery was obtained at 10:24 a.m. on May 1,2021, when the weather was clear and cloudless. This study used MicaSense Altum (Seattle, WA, USA) sensor installed on DJI Matrice200 four-axis aircraft. The MicaSense Altum has five high-resolution multispectral bands (blue, green, red, red edge, and near-infrared) and integrated long-wave thermal infrared (TIR) sensor (based on the FLIR Lepton), which is aligned with the multispectral sensors. The specific spectral parameters of the camera are shown in Table 1. The TIR sensor recalibrates every 5 min or when a $2 \mathrm{~K}$ change in temperature occurs. The reported accuracy is $+/-5 \mathrm{~K}$ with thermal sensitivity of $<50 \mathrm{mK}$. The flight altitude of the UAV is set at $30 \mathrm{~m}$, the heading overlap is $80 \%$, and the side overlap is $70 \%$. each field by stitching hundreds of different images captured during the same flight into one single 


\begin{tabular}{cccc}
\hline Band\# & Name & Center Wavelength(nm) & Bnadwidth(nm) \\
\hline 1 & Blue & 475 & 32 \\
2 & Green & 560 & 27 \\
3 & Red & 668 & 14 \\
4 & Red edge & 717 & 12 \\
5 & Near-infrared (NIR) & 842 & 57 \\
6 & Thermal infrared & Band Range $(\mu \mathrm{m}): 8-14$ \\
\hline
\end{tabular}

\section{Methods}

Figure 3 depicts the study workflow. The workflow was divided into two parts: feature extraction and LAI prediction model building and validation. Firstly, the UAV high spatial resolution dataset was divided into two datasets: the dataset to remove the soil background and the dataset to retain the soil background. Then the spectral, structural, and thermal features of the canopy were extracted, respectively. Secondly, the spectral, structural, and thermal features were fused, the machine learning methods (RFR and SVR) were used to model. Then the accuracy of the model was evaluated to find the best feature combination. Finally, the importance of each variable in the model was ranked based on the best feature combination. The common variables among the top ten variables were selected. MLR was used to model, and the accuracy of the model was evaluated. 

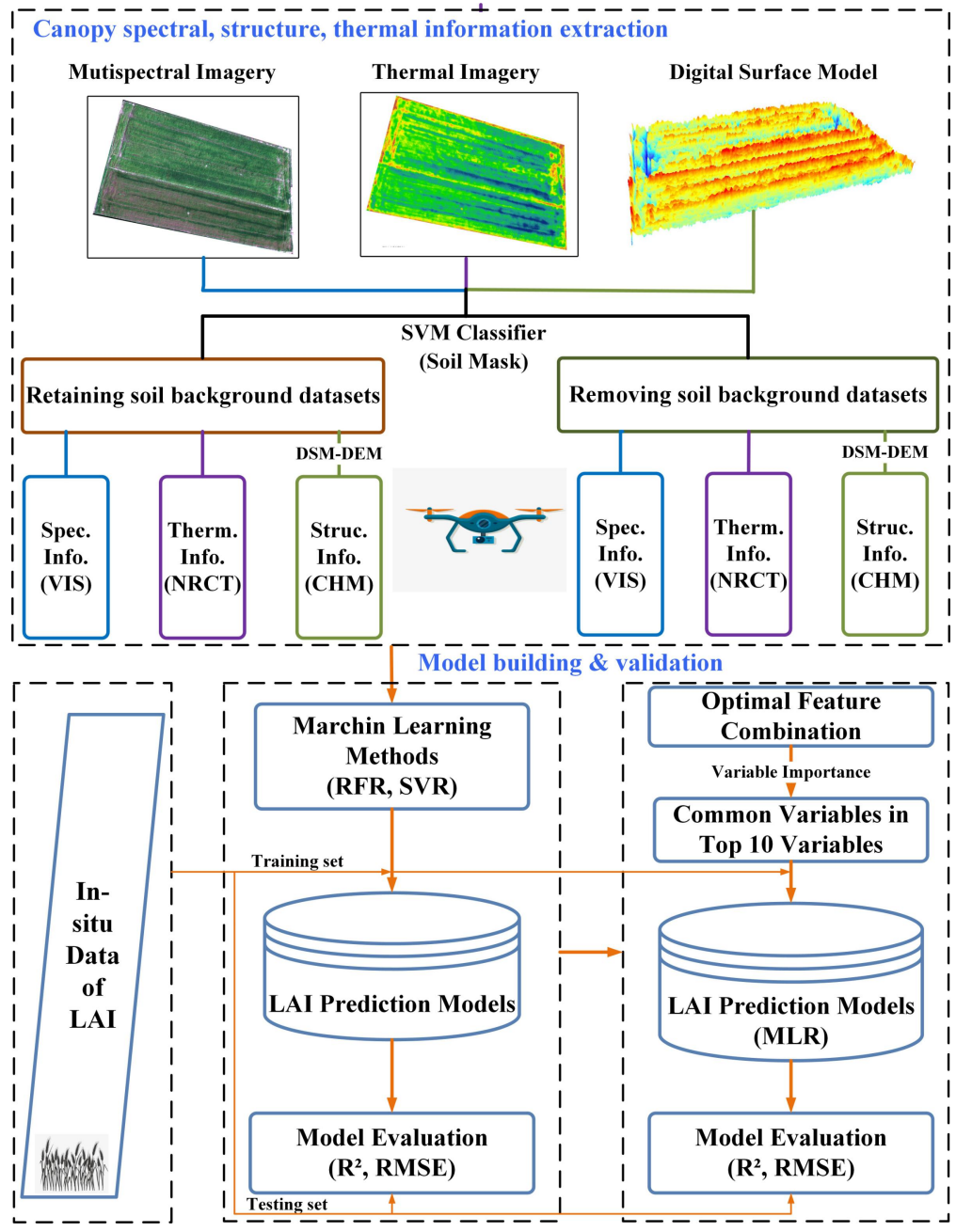

Fig. 3 A workflow diagram of data processing, feature extraction and modeling.

\section{Feature Extraction}

First, two datasets were prepared. For one dataset, the soil background was retained, which

172 means no background processing is done on dataset, referred to as the dataset with soil

173 background; for another dataset, the soil background was removed, referred to as the dataset

174 without soil background. The process of removing the soil background is as follows: display the

175 UAV multispectral imagery in the true color composite of the blue, green, and red bands. Support

176 Vector Machine (SVM) classifier was used to identify wheat and soil on the UAV imagery. A

177 binary mask layer was established to exclude background soil pixels from all spectral, structure, 
thermal, and texture features extracted from UAV imagery for further processing. The performance of the SVM classifier was evaluated using the confusion matrix and accuracy statistics overall accuracy (OA) and Kappa coefficient based on randomly selected independent test samples. The SVM classification resulted in an OA of $98.1 \%$ and a Kappa coefficient of 0.976 .
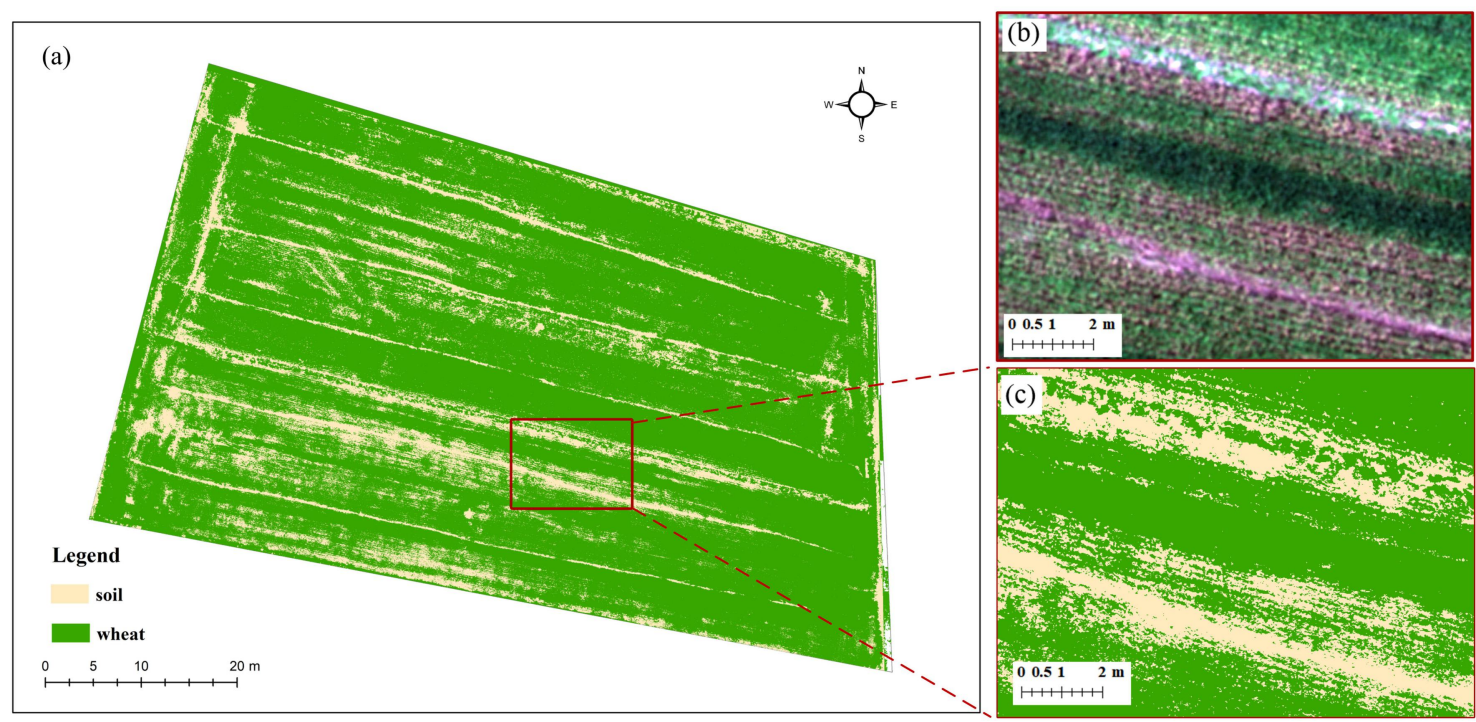

Fig. 3 Vegetation fraction and soil removal. (a) shows the entire field, (b) is a close-up RGB image, and (c) shows the corresponding vegetation and soil map of the close-up view.

For the two datasets with and without soil background, the following same processing is performed. Average pixel values for each of the spectral, structure, and thermal raster layers listed in Table 2 were computed at a small yield plot level using zonal statistics to relate them with the corresponding wheat LAI. The Arcpy library and Python 2.7 programming language were used to apply zonal statistics, remove soil background, and automate and streamline the extraction of raster layers, the extracted features as shown in Tab. 2.

Tab. 2 Definitions of the features extracted from different imagery.

\begin{tabular}{cllc}
\hline Feature & Features & Formulation & References \\
\hline Spec. & Blue(B), Green $(\mathrm{G}), \operatorname{Red}(\mathrm{R})$, Red-edge(RE), & The raw value of each band & $/$
\end{tabular}




\begin{tabular}{|c|c|c|c|}
\hline Info. & Near-infrared(NIR) & & \\
\hline & Ratio vegetation index & $\mathrm{RVI}=\mathrm{NIR} / \mathrm{R}$ & [31] \\
\hline & Green chlorophyll index & $\mathrm{GCI}=(\mathrm{NIR} / \mathrm{G})-1$ & [32] \\
\hline & Red-edge chlorophyll index & $\mathrm{RECI}=(\mathrm{NIR} / \mathrm{RE})-1$ & [32] \\
\hline & Normalized difference vegetation index & $\mathrm{NDVI}=(\mathrm{NIR}-\mathrm{R}) /(\mathrm{NIR}+\mathrm{R})$ & [33] \\
\hline & Green normalized difference vegetation index & GNDVI=(NIR-G)/(NIR+G) & [34] \\
\hline & Green-red vegetation index & $\mathrm{GRVI}=(\mathrm{G}-\mathrm{R}) /(\mathrm{G}+\mathrm{R})$ & [31] \\
\hline & Normalized difference red-edge & NDRE=(NIR-RE)/(NIR+RE) & [35] \\
\hline & Normalized difference red-edge index & $\mathrm{NDREI}=(\mathrm{RE}-\mathrm{G}) /(\mathrm{RE}+\mathrm{G})$ & [36] \\
\hline & Simplified canopy chlorophyll content index & $\mathrm{SCCCI}=\mathrm{NDRE} / \mathrm{NDVI}$ & [37] \\
\hline & Optimized soil adjusted vegetation index & OSAVI $=(\mathrm{NIR}-\mathrm{R}) /(\mathrm{NIR}+\mathrm{R}+\mathrm{L})(\mathrm{L}=0.16)$ & [38] \\
\hline & $\begin{array}{l}\text { Modified chlorophyll absorption in reflectance } \\
\text { index }\end{array}$ & MCARI $=[(\mathrm{RE}-\mathrm{R})-0.2 *(\mathrm{RE}-\mathrm{G})]^{*}(\mathrm{RE} / \mathrm{R})$ & [39] \\
\hline & $\begin{array}{l}\text { Transformed chlorophyll absorption in } \\
\text { reflectance index }\end{array}$ & $\mathrm{TCARI}=3 *[(\mathrm{RE}-\mathrm{R})-0.2 *(\mathrm{RE}-\mathrm{G}) *(\mathrm{RE} / \mathrm{R})]$ & [40] \\
\hline & MCARI/OSAVI & MCARI/OSAVI & [39] \\
\hline & TCARI/OSAVI & TCARI/OSAVI & [40] \\
\hline & Wide dynamic range vegetation index & WDRVI=(a*NIR-R $) /(a * N I R+R)(a=0.12)$ & [41] \\
\hline $\begin{array}{l}\text { Struc. } \\
\text { Info. }\end{array}$ & Canopy Height Model (m) & $\mathrm{CHM}=\mathrm{DSM}-\mathrm{DEM}$ & l \\
\hline $\begin{array}{l}\text { Therm. } \\
\text { Info. }\end{array}$ & Normalized relative canopy temperature index & $N R C T=\frac{T_{i}-T_{\min }}{T_{\max }-T_{\min }}$ & [42] \\
\hline
\end{tabular}

\section{Canopy Spectral Information} monitoring and trait estimation as usual, such as NDVI, RVI, and NDRE, were selected (Tab. 2).

\section{Canopy Structure Information}


the ground and all objects on the ground, while DEM only represents the height of the ground, the CHM could be obtained by subtracting DEM from DSM. An essential step in creating DEM and DSM is to identify Ground Points (GP) and Non-Ground Points (NGP) in dense point clouds, which is performed in Pix4Dmapper software. This tool allows distinguishing between GP, NGP, and noise points. DSM is created from all categories except noise points, while DTM is calculated only from points identified as GP.

\section{Canopy Thermal Information}

To facilitate the use of temperature data, it is normalized and mapped to the range of $0-1$. The thermal feature of LAI prediction, the Normalized Relative Canopy Temperature (NRCT) [42], was calculated from thermal infrared images. NRCT was computed using the canopy temperature in the imagery, the minimum temperature ( $\mathrm{T}_{\min }$, lower baseline), and the maximum temperature ( $T_{\max }$, upper baseline) of the whole study area, as shown in Figure 4. NRCT has been used to evaluate water status and crop traits. The larger the value of NRCT, the higher the temperature; the smaller the value of NRCT, the lower the temperature $[42,45]$. The NRCT was calculated based on the following equation:

$$
N R C T=\frac{T_{i}-T_{\min }}{T_{\max }-T_{\min }}
$$

where $T_{i}$ represents the canopy temperature of the $i$ th pixel, $T_{\min }$ is the lowest temperature in the whole field trial, and $T_{\max }$ the highest temperature in the whole field trial 


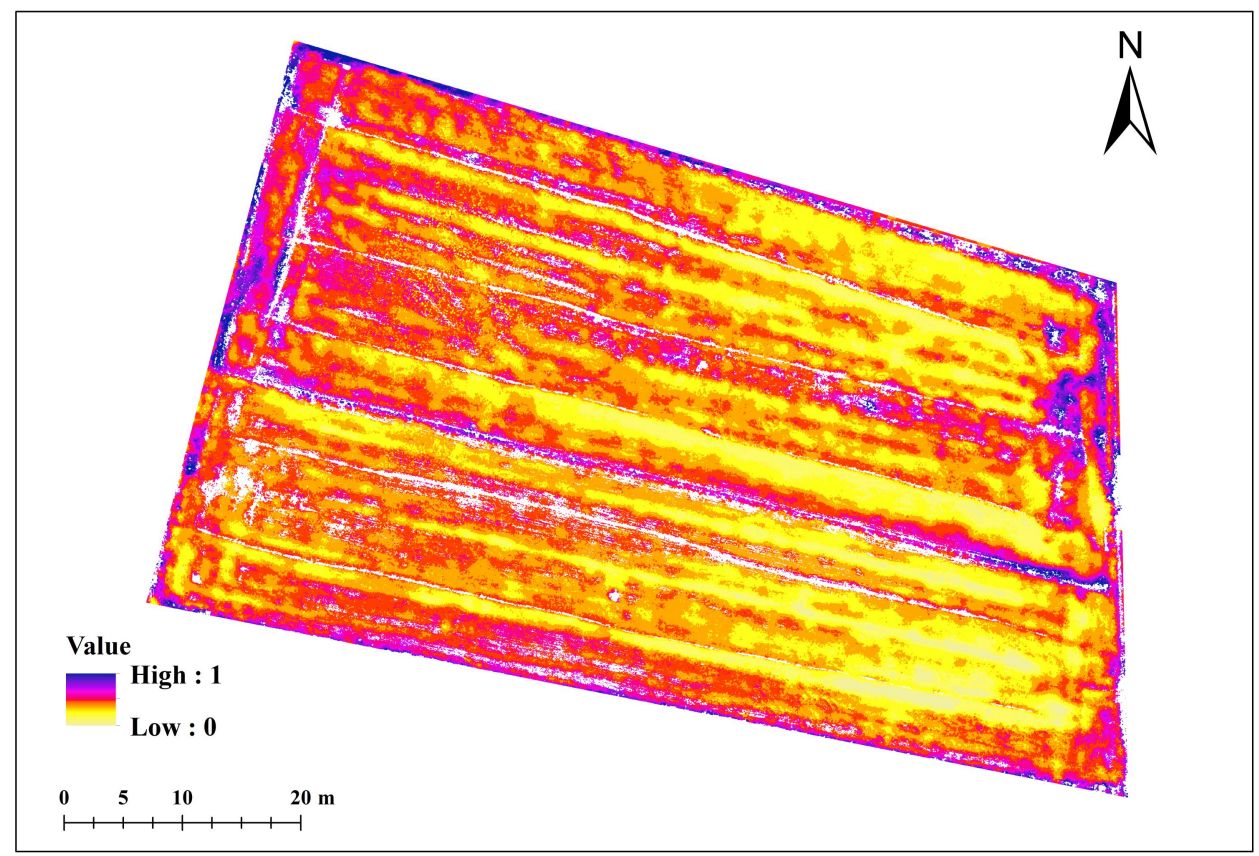

Fig. 4 Normalized relative canopy temperature index distribution map (removed soil pixels are represented

by white color).

\section{LAI Prediction Model Building and Validation}

Several machine learning methods were used in remote sensing applications, especially in crop monitoring and trait estimation, such as RFR and SVR. This study used RFR and SVR to estimate the LAI based on canopy spectrum, structure, and thermal features. RFR is a nonparametric integration method based on the Classification and Regression Tree (CART). It is made up of different trees that have been trained using bagging and random variable selection,

230 which is more tolerant of outliers and noise [46,47]. The number of decision trees determines the 231 performance of RFR. The number of decision trees tested was 50, 100, 150, and 200. To strike a

232 balance between calculation time and accuracy, the number of decision trees was finally set at 100 .

233 SVR is a form of nonparametric modeling that defines boundaries in a high-dimensional space 
using a hyperplane [48,49]. To construct an SVR with good performance, the selection of kernel function is the key, so the linear kernel function is selected [48].

First, multiple features are fused, and then the fused features are used as input features of the model. To better train and evaluate the model, $70 \%$ randomly selected input features and LAI were used as training samples, and the remaining $30 \%$ were used as unseen samples to test the performance of the model. The coefficients of determination $\left(\mathrm{R}^{2}\right)$ and root mean square error (RMSE) were computed to evaluate the performance of the LAI prediction model and can be expressed as follows:

where $y_{i}$ and $\hat{y}_{i}$ are the measured and the predicted LAI, respectively. $\bar{y}$ is the mean of measured LAI, and $n$ is the total number of samples in the testing set.

To estimate LAI quickly and efficiently, the best feature combination was determined by comparing the model's accuracy. For the best feature combination, according to the importance of variables in the RFR and SVR models, common variables were found in the top ten variables of the two models. And then, MLR was to build the model based on common variables. It is known that the MLR is to predict or estimate the dependent variable by the optimal combination of multiple independent variables, which is more effective and practical than using only one independent variable [50]. The IBM SPSS Modeler 18.0 was used to build the LAI prediction model and variable importance ranking. 
involving variables and a response variable depending on linear equation into the observed data.

256 Compared with most regression methods of machine learning, it is simpler and easier to operate.

The MLR model is:

$$
y_{i}=b_{0}+b_{1} x_{i, 1}+b_{2} x_{i, 2}+\ldots+b_{k} x_{i, k}+e_{i}
$$

Where, $y_{i}$ is the dependent variable; $\mathrm{b}_{0}$ is the intercept; $x_{i, k}$ is an independent variables; $b_{k}$ is

the vector of regression coefficients; and $e_{i}$ is random measured errors.

\section{Results}

\section{Correlation between LAI and Each Variable}

Features were extracted from datasets with and without soil background to investigate the and the results are shown in Figure 5.
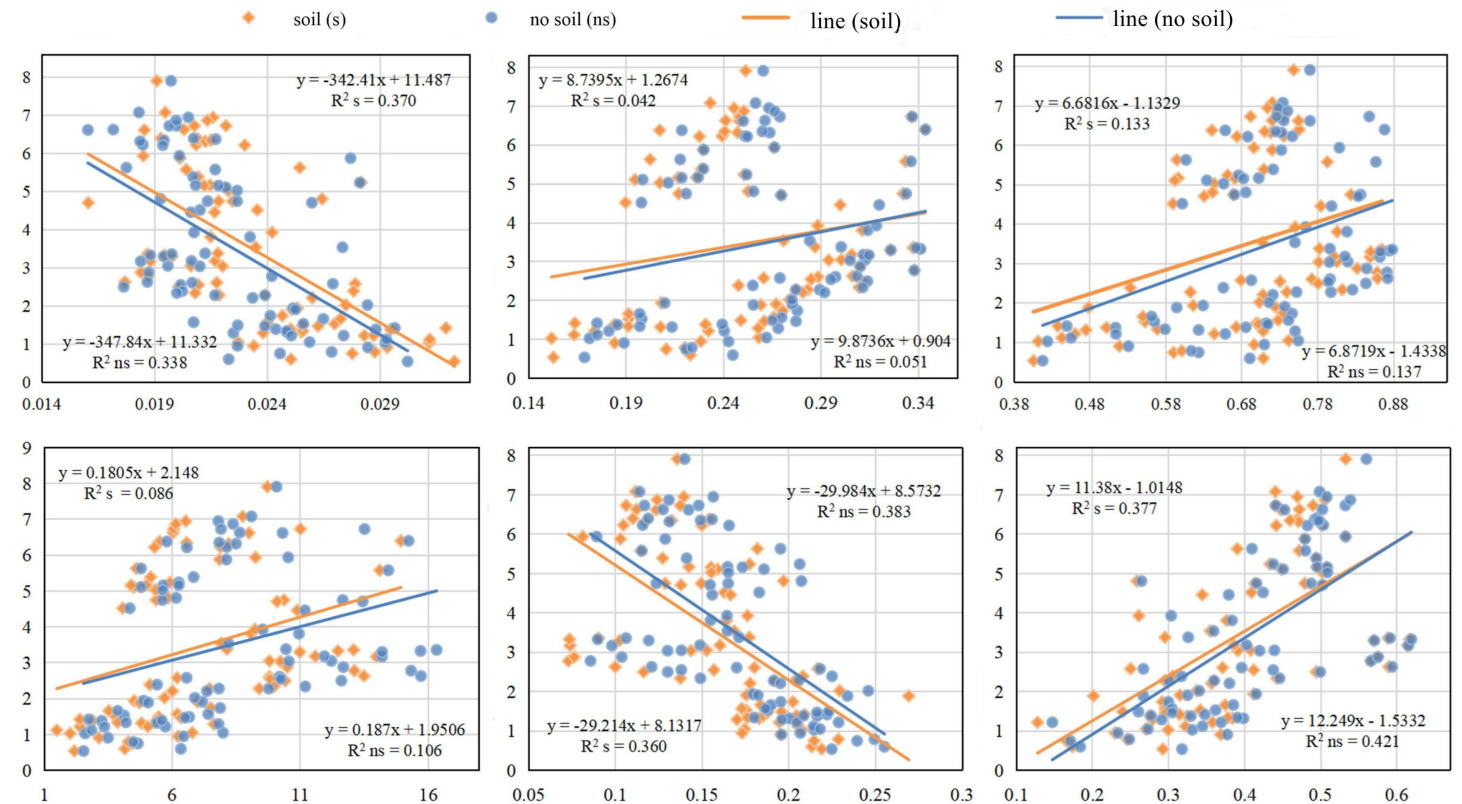

Fig. 5 Correlation between LAI and various features with or without soil background. Ns and s denote the 
276 features is not high whether there is soil background or not.

277

\begin{tabular}{cccccc}
\hline \multirow{2}{*}{ Feature } & Metrics & \multicolumn{2}{c}{ Removing soil background } & \multicolumn{2}{c}{ Retaining soil background } \\
\cline { 3 - 6 } & & RFR & SVR & RFR & SVR \\
\cline { 3 - 6 } Sp & $\mathrm{R}^{2}$ & 0.746 & 0.679 & 0.684 & 0.539 \\
& $\mathrm{RMSE}$ & 1.185 & 1.233 & 1.441 & 1.472 \\
$\mathrm{~S} \mathrm{Sp}+\mathrm{Th}$ & $\mathrm{R}^{2}$ & 0.689 & 0.701 & 0.573 & 0.536 \\
& $\mathrm{RMSE}$ & 1.391 & 1.176 & 1.604 & 1.474 \\
$\mathrm{Sp}+\mathrm{St}$ & $\mathrm{R}^{2}$ & 0.792 & 0.741 & 0.773 & 0.584 \\
& $\mathrm{RMSE}$ & 1.135 & 1.127 & 1.135 & 1.391 \\
$\mathrm{Sp}+\mathrm{Th}+\mathrm{St}$ & $\mathrm{R}$ & 0.815 & 0.748 & 0.781 & 0.576 \\
& $\mathrm{RMSE}$ & 1.023 & 1.121 & 1.128 & 1.304 \\
\hline
\end{tabular}

(Sp represents spectral features, St represents structure features, Th represents thermal features. The darker the the accuracy of retaining the soil background.)

RFR and SVR were employed for LAI prediction using canopy spectral, structure, and thermal features with or without soil background, respectively, the validation statistics of different models in Table 3.

Tab. 3 Validation statistics of different models for wheat LAI prediction.

blue, the higher the accuracy of the model for removing the soil background, and the darker the yellow, the higher Firstly, the features extracted from the dataset without soil background are analyzed. It can be

\section{LAI Prediction Model}


seen that when only spectral features are used, the range of $\mathrm{R}^{2}$ is $0.679-0.746$ and the range of RMSE is 1.233-1.185. Compared with the blue or near-infrared band used alone in the previous section, $\mathrm{R}^{2}$ is increased by 0.25 or 0.62 . It demonstrates that fusing all spectral features is superior to using any spectral feature alone, and the accuracy has been significantly improved.

When compared to spectral features alone, combining spectral and thermal features slightly improve the accuracy of the SVR model ( $\mathrm{R}^{2}: 0.701$ and RMSE: 1.176$), \mathrm{R}^{2}$ increases by 0.03 , and RMSE decreases by 0.057 . The prediction accuracy of the RFR model decreases $\left(\mathrm{R}^{2}: 0.689\right.$ and RMSE: 1.391$), \mathrm{R}^{2}$ decreases by 0.11 , and RMSE increases by 0.206 . It is demonstrated that the combination of spectral and thermal features performs poorly in the LAI prediction model, as shown in Figure 6.

When combining structural and spectral features, the prediction accuracy of both RFR and SVR models is improved compared with the spectral features alone and the combination of spectral and thermal features. Compared with spectral features alone, $\mathrm{R}^{2}$ of the RFR model increases by 0.05 and RMSE decreases by $0.05 ; \mathrm{R}^{2}$ of the SVR model increases by 0.07 , and RMSE decreases by 0.106 . Compared with the combination of spectral and thermal features, $\mathrm{R}^{2}$ of the RFR model increases by 0.11 and RMSE decreases by $0.256, \mathrm{R}^{2}$ of the SVR model increases by 0.04 , and RMSE decreases by 0.049 . 

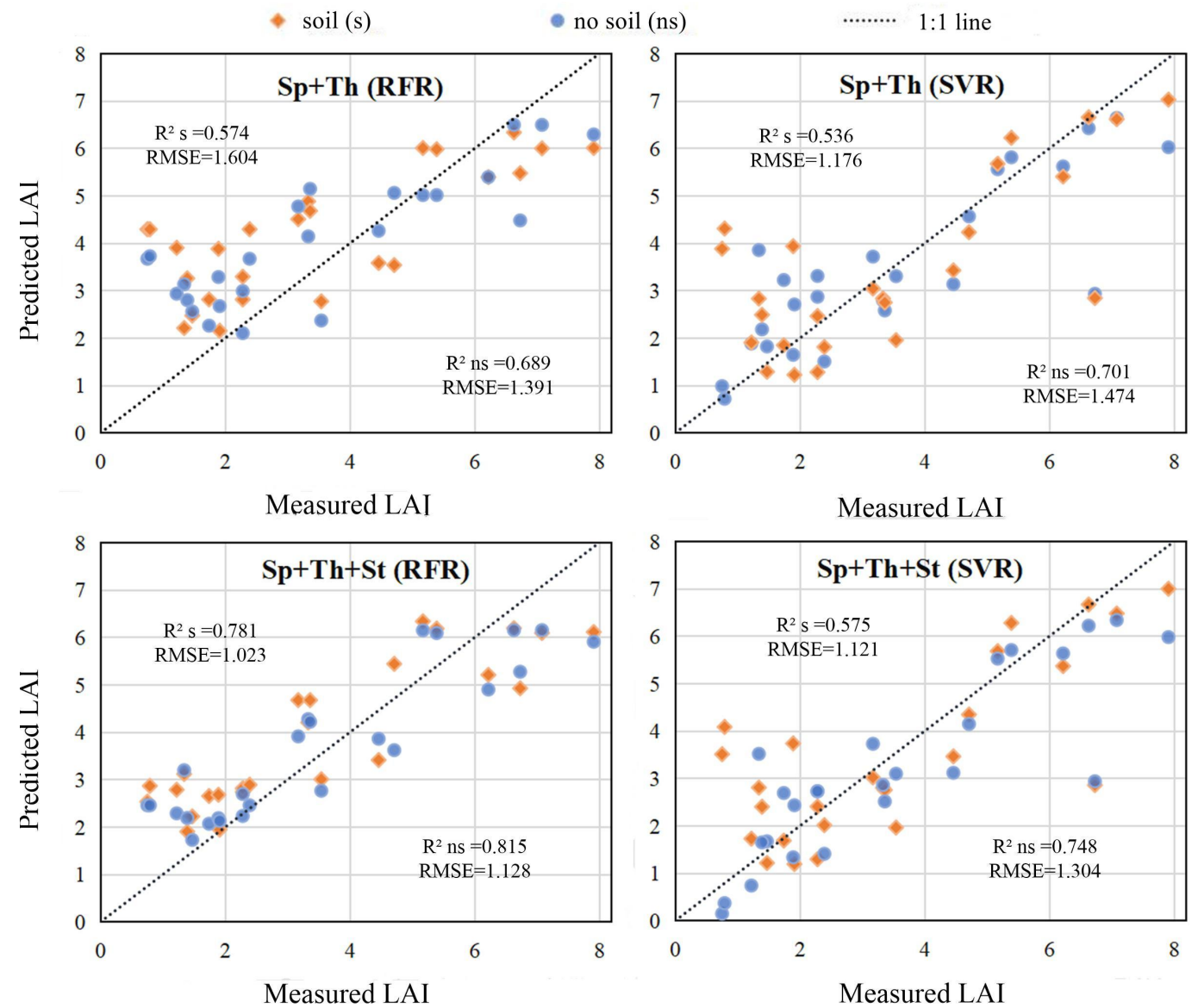

Fig. 6 The cross-validation scatter plots for measured versus prediction LAI.

When all features were added to the model, the model's prediction accuracy improves to some extent, and the accuracy peaks $\left(\mathrm{R}^{2}: 0.748-0.815\right.$ and RMSE: 1.023-1.121), as shown in Fig. 6. $\mathrm{R}^{2}$ increases by 0.069 , and RMSE decreases by 0.162 compared to the RFR model using only spectral feature; $\mathrm{R}^{2}$ increases by 0.069 , and RMSE decreases by 0.112 compared to the SVR model using only spectral feature. When combining spectral and thermal features, $\mathrm{R}^{2}$ increases by 0.126 , and RMSE decreases by 0.368 for the RFR model; for the SVR model, $\mathrm{R}^{2}$ increases by 0.047, while RMSE decreases by 0.055 . Compared to the combination of spectral and structural

312 features, $\mathrm{R}^{2}$ increased by 0.023 and RMSE decreases by 0.112 for the RFR model, $\mathrm{R}^{2}$ increases by

3130.007 , and RMSE decreases by 0.006 for the SVR model. It can be seen that the model accuracy of the combination of all features is not improved compared to the model of the combination of 
spectral and structural features, indicating that adding thermal features to spectral and structural features has little effect on LAI prediction accuracy. Simultaneously, it is demonstrated that combining multiple features is superior to using only one or two features.

Similar to the model without soil background, the model's prediction accuracy with soil background is reduced by combining thermal and spectral features compared to the combination of spectral features. Combining spectral and structural features improves the model's accuracy using only spectral features ( $\mathrm{R}^{2}: 0.584-0.773$ and RMSE: 1.135-1.391). Secondly, for the RFR model, the prediction accuracy of combining spectral, structural, and thermal features reaches the highest $\left(\mathrm{R}^{2}=0.748\right.$, and $\left.\mathrm{RMSE}=1.128\right)$

However, the model with soil background was different from the model without soil background. Whether RFR or SVR was used, the model without soil background was higher ( $\mathrm{R}^{2}: 0.679-0.746$ and RMSE:1.185-1.233). Similarly, for the combination of spectral and thermal features and the combination of spectral and structural features, the model without soil background has higher accuracy than the model with soil background $\left(\mathrm{R}^{2}: 0.689-0.701\right.$, RMSE:1.176-1.391, and $\mathrm{R}^{2}:$ 0.741-0.746, RMSE:1.135-1.127). When combining spectral, structure, and thermal features, compared with the model with soil background, the $\mathrm{R}^{2}$ of the RFR model without soil background increases from 0.781 to 0.815 , and the RMSE decreases from 1.128 to 1.023 ; for the SVR model, $\mathrm{R}^{2}$ increases from 0.576 to 0.748 and RMSE decreases from 1.304 to 1.121 . It is shown that the soil background can be removed well with the help of high-resolution UAV imagery. This operation retains the pure vegetation characteristics, avoids the interference of soil factors on the model, and improves the prediction accuracy of LAI. 


\section{The Importance of Variables}

It can be seen that the highest prediction accuracy can be obtained based on the combination of spectral, structural, and thermal features without soil background. The importance of variables in RFR and SVR models was ranked based on the best fusion features, as shown in Figure 7.
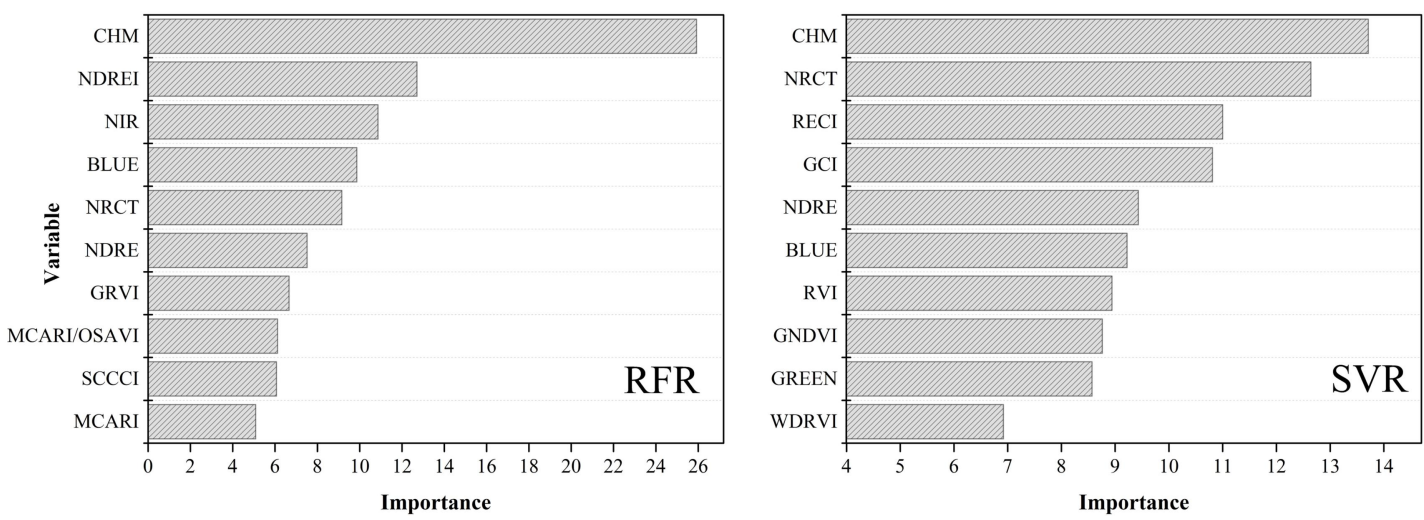

Fig. 7 Top 10 features in importance.

It could be seen that the score of CHM is the highest among the importance of variables in the RFR and SVR models, indicating that the structural feature plays an essential role in both models (Fig. 7). It is due to the structural feature being relatively independent of the other features in the model and contributing the most to the LAI estimation. In addition to the CHM, which has the highest importance score in the RFR model, NDREI, NIR, BLUE, and NRCT have higher importance scores. NRCT, RECI, GCI, and NDRE have higher importance scores except for CHM in the SVR model. The importance scores of thermal features and some VIs are high relatively, indicating that they performed well in LAI estimation.

It can be found that there are four common variables in the top ten variables of the two models, namely CHM, BLUE, NRCT, and NDRE. To quickly estimate the LAI of wheat, MLR was used based on these four common variables, and the multivariate linear formula was as follows. 


$$
y=-390.65 * x_{B L U E}-12.14 * x_{N D R E}-3.2 * x_{N R C T}+9.49 * x_{C H M}+12.92
$$

To better evaluate the effect of the MLR model in estimating LAI, RFR and SVR models are used simultaneously. The results are shown in Table 4.

Tab. 4 Verification accuracy of different regression methods.

\begin{tabular}{cccc}
\hline CHM+NRCT+BLUE+NDRE & MLR & RFR & SVR \\
\hline $\mathrm{R}^{2}$ & & & 0.734 \\
$\mathrm{RMSE}$ & 0.679 & 1.156 & 1.413 \\
\hline
\end{tabular}

It can be seen that the prediction accuracy of the SVR model based on four common

variables is the lowest $\left(\mathrm{R}^{2}=0.584\right.$ and RMSE $\left.=1.413\right)$, and the prediction accuracy of the RFR model is the highest $\left(\mathrm{R}^{2}=0.584\right.$ and RMSE $\left.=1.156\right)$. High prediction accuracy can be obtained using the MLR model predicted LAI of four variables $\left(\mathrm{R}^{2}=0.679\right.$ and RMSE $\left.=1.231\right)$. In addition, compared with the prediction accuracy of the RFR model $\left(\mathrm{R}^{2}=0.815\right.$ and $\mathrm{RMSE}=$ 1.023 $)$ and SVR model $\left(\mathrm{R}^{2}=0.748\right.$ and RMSE $\left.=1.121\right)$ using all the features without soil background, the prediction accuracy obtained by MLR is little different. Although the prediction accuracy of the MLR model is slightly lower than that of the RFR model, MLR is more straightforward, easier to operate, and shorter than RFR. It is demonstrated that to some extent, using four variables (CHM, BULE, NRCT, and NDRE), the MLR model can replace the RFR model using all features. It is also demonstrated that using fewer variables instead of all variables could achieve higher LAI prediction accuracy, reduce the system calculation time, and improve obtained, as shown in Figure 8. 


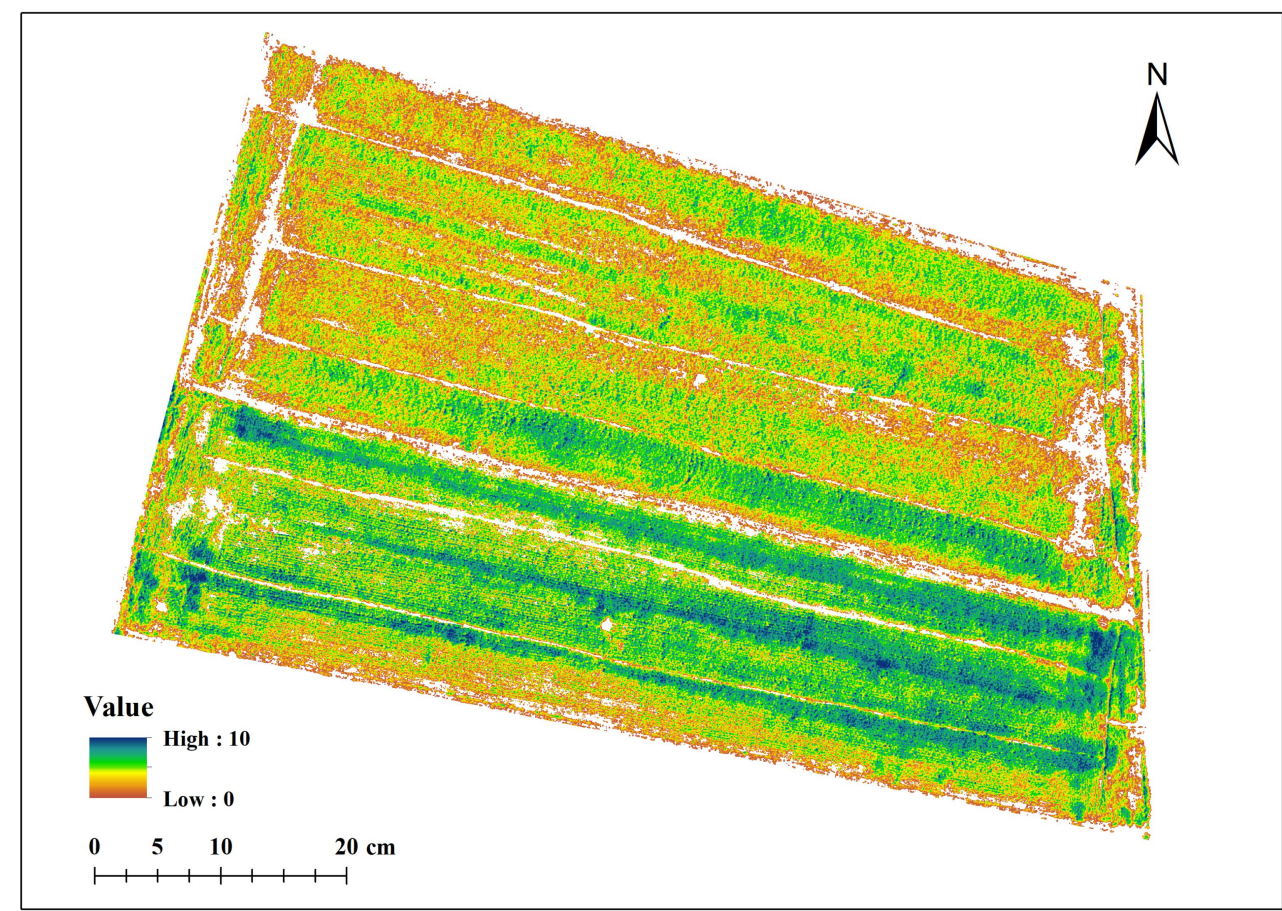

Fig. 8 LAI prediction map derived when applying the MLR model to the 4 common variable images.

\section{Discussion}

\section{Influence of High-Resolution UAV Image}

Previous research has shown that removing pure background pixels in high-resolution UAV imagery can improve crop trait estimation accuracy. Shu et al. [17] discovered that removing the soil background from $1.9 \mathrm{~cm} /$ pixel UAV hyperspectral imagery can effectively estimate the SPAD value of corn leaves. This study used $1.49 \mathrm{~cm} /$ pixel UAV multispectral imagery to improve the prediction accuracy of wheat LAI without soil background, which is essential for wheat field management and precision agriculture. Furthermore, it is widely assumed that the influence of soil background on vegetation-soil systems has two components [11]. One factor is that soil components derived from mixed vegetation and soil pixels directly impact vegetation reflectance. The other factor is that multi-scattering between soil and vegetation indirectly affects the vegetation spectrum, which is more complex. We will further consider the impact of soil factors on vegetation spectral information in the future. 

combined with multi-sensor synchronous observation on LAI estimation, only UAV imagery with the high resolution of $1.49 \mathrm{~cm} /$ pixel is used for research. Previous research has shown that images with different spatial resolutions have different effects on the accuracy of crop trait estimation. For example, Guo et al. [51] used different flight altitudes to evaluate the impact of UAV images with different spatial resolutions on SPAD prediction. It was found that compared with images obtained with flight altitudes of $75 \mathrm{~m}(2.1 \mathrm{~cm} /$ pixel $), 100 \mathrm{~m}(2.8 \mathrm{~cm} / \mathrm{pixel})$, and $125 \mathrm{~m}(3.4 \mathrm{~cm} /$ pixel $)$, imagery with a flying height of $50 \mathrm{~m}$ (spatial resolution of $1.8 \mathrm{~cm} / \mathrm{pixel}$ ) can be used to estimate SPAD in leaves accurately. To further investigate the impact of UAV images with different spatial resolutions on LAI prediction, we resampled the image into the $5 \mathrm{~cm} /$ pixel UAV images without soil background. After combining all the features, RFR was used to predict LAI. Compared with $\mathrm{cm} /$ pixel imagery modeling decreased by about $0.3-0.4$, and the RMSE increased by about $2-3$. It

\section{Contribution of Different Feature}


overcome the problem of asymptotic saturation inherent in spectral features [55]. Second, using a combination of spectral, structural, and thermal features to estimate LAI yielded the highest accuracy, which is consistent with previous research [25]. The results show that the canopy's spectral, structural, and thermal features can provide unique and complementary information that can be used to predict LAI.

Previous research has shown that when all other factors are held constant, the greater the leaf area per unit surface area, or the greater the LAI value, the greater the water content of the crop itself $[56,57]$. Because thermal features are related to leaf water content, pigment concentration, and canopy structure, LAI is closely associated with thermal features [58-60]. However, complex environmental conditions such as soil background, water availability, and atmospheric conditions all impact canopy temperature [61]. As a result, in this study, spectral and thermal features were used as input parameters of the model. Whether RFR or SVR was used, the accuracy will be reduced to some extent. This phenomenon could be caused by environmental factors such as average temperature, soil moisture, and organic matter composition in soil. In the follow-up research, it is necessary to further understand the relationship between canopy thermal characteristics and LAI, especially under various factors such as different plant species, development stages, environmental conditions, and interactions. It should be noted that adding thermal features to the spectral features reduced the accuracy. However, when ranking the importance of variables in the model after combining spectral, structural, and thermal features, it is clear that thermal features have high significance. This phenomenon could be caused by the interaction of spectral, thermal, and structural features, emphasizing the importance of NRCT. Furthermore, NRCT was used in this study to quantify temperature. NRCT has been used to assess 
and forecast crop water status and traits. In general, NRCT requires the measured ground temperature to normalize. However, the canopy temperature is not counted due to external factors (environment and equipment, for example). Still, the thermal features are obtained directly by using statistical values in the thermal infrared image of the UAV. The measured ground temperature data will be used in the follow-up study to predict wheat LAI better.

\section{Uncertainty and Outlook}

Since the purpose of this study is to investigate the application potential of high spatial resolution of UAV imagery combined with multi-sensor synchronous observation in LAI estimation, RFR and SVR are used for all spectral, structural, and thermal features combinations. Of course, MLR is also used to estimate LAI, but due to the poor effect, it is not shown in this article; only the RFR and SVR methods with the best impact are shown. However, for a few essential features selected from all variables. The MLR method achieves satisfactory results, shortens the running time of the model, improves the efficiency, and provides an idea for rapid estimation of LAI of wheat.

In the past decade, the performance of deep learning developed from traditional neural networks has been significantly improved, surpassing the traditional models in the field of earth observation [62]. However, it has certain limitations, for example, a large number of training data are needed to effectively converge the deep learning model to obtain the optimal model parameters. Shallow machine learning regression models, which include RFR, SVR, ANN, do not need a lot of training data. Compared with traditional algorithms, the machine learning regression model can effectively use data when dealing with high-dimensional and complex data to obtain higher prediction accuracy of the model $[18,63]$. Because the purpose of this paper is to 
investigate the impact of different feature fusion on LAI prediction under high-resolution UAV imagery, only three machine learning methods (RFR, SVR, and MLR) were used. In addition, this study found that no matter which features fusion is used, RFR performs best in LAI prediction, consistent with previous studies on other traits of crops [18,64]. Zha et al. [64] found RF performed better than SVR, MLR, and ANN on predicting nitrogen content in rice using spectral features. Previous studies have also shown that SVR is a new generation of machine learning algorithms based on statistical learning theory. The advantages of SVR are mainly reflected in solving linear inseparable problems. It solves the inner product operation in high-dimensional space by introducing kernel function to solve the nonlinear problem [48,49]. The kernel functions of SVR include radial basis function, polynomial, sigmoid kernel, and linear kernel function. Because of many experiments and the advantages of fewer parameters and fast speed, the linear kernel function with the best performance was selected to predict the LAI of wheat.

Previous research has shown that studying data from the entire crop growth cycle can help us determine which period can accurately predict crop traits and growth in the early stages [19]. However, this study only included wheat at the heading stage as the study object due to the weather and crew availability. In future research, we will consider the prediction of LAI throughout the wheat growth cycle.

\section{Conclusion}

Under the data fusion framework of UAV high-resolution imagery and multi-sensor synchronous observation, the influence of soil background on LAI estimation was investigated. The following conclusions could be drawn: (1) the soil background will affect the estimation of wheat LAI. The high-resolution UAV imagery can effectively remove the soil background, and the 
prediction accuracy is significantly improved after removal. (2) Fusing multiple data (spectral, structural, and thermal features) observed synchronously by multiple sensors significantly improves the prediction accuracy than using one or two data to estimate LAI and achieves the highest accuracy $\left(\mathrm{R}^{2}=0.815\right.$ and RMSE $\left.=1.023\right)$. (3) High prediction accuracy can be achieved by using a simple model based on CHM, NRCT, NDRE, and BLUE, combined with multiple linear regression to estimate LAI.

The finding indicates that the method based on UAV high-resolution imagery combined with multi-sensor data fusion has a great potential for crop LAI estimation. Furthermore, it has guiding significance for high-precision LAI rapid prediction using UAV remote sensing technology and a reference value for precision agriculture promotion and application. However, to further assess its robustness, this method will need to be tested on different crop types at different stages of development and under different environmental conditions in future research.

\section{Acknowledgement}

The authors are very thankful for Hanyue Zou, Tianxing Fan, Kongbo Wang and Zhuo Lu for their valuable support.

\section{Author's Contributions}

S.W., L.G. and L.D. designed and developed the research idea. L.G., Y.W. and S.W. conducted the field data collection. S.W., L.D., L.G. and Y.W. processed all remaining data. S.W. and L.G. performed the data analysis and wrote the manuscript. S.W., L.D., L.G. and Y.W. contributed to result and data interpretation, discussion, and revision of the manuscript. All authors have read and agreed to the published version of the manuscript.

\section{Funding}

This research was funded by Based on Multi-Scale High Resolution Remote Sensing Technology Project Grant number 21220010040. 
501 The remotely sensed and field sampling data used in this study is available from the corresponding

502 author on reasonable request.

\section{Declarations}

Ethics approval and consent to participate

All authors read and approved the manuscript.

\section{7}

\section{Consent for publication}

All authors agreed to publish this manuscript.

\section{Competing interests}

The authors declare that they have no known competing financial interests or personal relationships that could have appeared to influence the work reported in this paper.

\section{Author details}

*Correspondence: denglei@cnu.edu.cn

${ }^{1}$ College of Resource Environment and Tourism, Capital Normal University, Beijing 100048, China. ${ }^{2}$ Engineering Research Center of Spatial Information Technology, Ministry of Education, Capital Normal University, Beijing 100048, China. ${ }^{3}$ Beijing Laboratory of Water Resources Security, Capital Normal University, Beijing 100048, China

\section{References:}

1. Qiu B, Luo Y, Tang Z, et al. Winter wheat mapping combining variations before and after estimated heading dates. ISPRS J PHOTOGRAMM 2017;123:35-46

2. Nasrallah A, Baghdadi N, El Hajj M, et al. Sentinel-1 Data for Winter Wheat Phenology Monitoring and Mapping. REMOTE SENS-BASEL 2019;11:2228

3. Zhong L, Hu L, Zhou H, Tao X. Deep learning based winter wheat mapping using statistical data as ground references in Kansas and northern Texas, US. REMOTE SENS ENVIRON 2019;233:111411

4. Liu X, Jin J, Herbert SJ, Zhang Q, Wang G. Yield components, dry matter, LAI and LAD of soybeans in Northeast China. FIELD CROP RES 2005;93:85-93

5. Zhou X, Zheng HB, Xu XQ, et al. Predicting grain yield in rice using multi-temporal vegetation indices from UAV-based multispectral and digital imagery. ISPRS J PHOTOGRAMM 2017;130:246-255 

Piecewise LAI-VI Relationships Based on Phenophases. REMOTE SENS-BASEL 2019;11:689

7. Goude M, Nilsson U, Holmström E. Comparing direct and indirect leaf area measurements for Scots pine and Norway spruce plantations in Sweden. EUR J FOREST RES 2019;138:1033-1047

8. Yan $\mathrm{G}, \mathrm{Hu}$ R, Luo J, et al. Review of indirect optical measurements of leaf area index: Recent advances, challenges, and perspectives. AGR FOREST METEOROL 2019;265:390-411

9. Weissa M, Jacobb F, Duveillerc G. Remote sensing for agricultural applications: A meta-review. REMOTE SENS ENVIRON 2020;236:111402

10. WATSON DJ. Comparative Physiological Studies on the Growth of Field Crops. ANN BOT-LONDON 1947;11:41-76

11. Yu K, Lenz-Wiedemann V, Chen X, Bareth G. Estimating leaf chlorophyll of barley at different growth stages using spectral indices to reduce soil background and canopy structure effects. ISPRS J PHOTOGRAMM 2014;97:58-77

12. Herrmann I, Pimstein A, Karnieli A, et al. LAI assessment of wheat and potato crops by VEN $\mu \mathrm{S}$ and Sentinel-2 bands. REMOTE SENS ENVIRON 2011;115:2141-2151

13. Kamenova I, Dimitrov P. Evaluation of Sentinel-2 vegetation indices for prediction of LAI, fAPAR and fCover of winter wheat in Bulgaria. EUR J REMOTE SENS 2021;54:89-108 14. Meyer LH, Heurich M, Beudert B, Premier J, Pflugmacher D. Comparison of Landsat-8 and Sentinel-2 Data for Estimation of Leaf Area Index in Temperate Forests. REMOTE SENS-BASEL 2019;11:1160

15. Mathews A, Jensen J. Visualizing and Quantifying Vineyard Canopy LAI Using an Unmanned Aerial Vehicle (UAV) Collected High Density Structure from Motion Point Cloud. Remote sensing (Basel, Switzerland) 2013;5:2164-2183

16. Zhang S, Zhao G, Lang K, et al. Integrated Satellite, Unmanned Aerial Vehicle (UAV) and

556 Ground Inversion of the SPAD of Winter Wheat in the Reviving Stage. SENSORS-BASEL 2019;19:1485

17. Shu M, Zuo J, Shen M, et al. Improving the estimation accuracy of SPAD values for maize leaves by removing UAV hyperspectral image backgrounds. INT J REMOTE SENS 2021;42:5862-5881 18. Lee H, Wang J, Leblon B. Using Linear Regression, Random Forests, and Support Vector Machine with Unmanned Aerial Vehicle Multispectral Images to Predict Canopy Nitrogen Weight in Corn. REMOTE SENS-BASEL 2020;12:2071 19. Yang K, Gong Y, Fang S, et al. Combining Spectral and Texture Features of UAV Images for the Remote Estimation of Rice LAI throughout the Entire Growing Season. REMOTE SENS-BASEL 2021;13:3001

20. Maimaitijiang M, Sagan V, Sidike P, et al. Soybean yield prediction from UAV using multimodal data fusion and deep learning. REMOTE SENS ENVIRON 2020;237:111599 21. Schirrmann M, Giebel A, Gleiniger F, et al. Monitoring Agronomic Parameters of Winter Wheat Crops with Low-Cost UAV Imagery. REMOTE SENS-BASEL 2016;8:706 
Photography from Model Aircraft for Remote Sensing of Crop Biomass and Nitrogen Status. PRECIS AGRIC 2005;6:359-378

23. Primicerio J, Di Gennaro SF, Fiorillo E, et al. A flexible unmanned aerial vehicle for precision agriculture. PRECIS AGRIC 2012;13:517-523

24. Simpson JE, Holman F, Nieto H, et al. High Spatial and Temporal Resolution Energy Flux Mapping of Different Land Covers Using an Off-the-Shelf Unmanned Aerial System. REMOTE SENS-BASEL 2021;13:1286

25. Rischbeck P, Elsayed S, Mistele B, et al. Data fusion of spectral, thermal and canopy height parameters for improved yield prediction of drought stressed spring barley. EUR J AGRON 2016;78:44-59

26. Fieuzal R, Marais Sicre C, Baup F. Estimation of corn yield using multi-temporal optical and radar satellite data and artificial neural networks. INT J APPL EARTH OBS 2017;57:14-23

27. Aghighi H, Azadbakht M, Ashourloo D, Shahrabi HS, Radiom S. Machine Learning Regression Techniques for the Silage Maize Yield Prediction Using Time-Series Images of Landsat 8 OLI. IEEE J-STARS 2018;11:4563-4577

28. Swatantran A, Dubayah R, Goetz S, et al. Mapping migratory bird prevalence using remote sensing data fusion. PLOS ONE 2012;7:e28922

29. Maimaitijiang M, Sagan V, Sidike P, et al. Crop Monitoring Using Satellite/UAV Data Fusion and Machine Learning. REMOTE SENS-BASEL 2020;12:1357

30. Jin Z, Azzari G, Lobell DB. Improving the accuracy of satellite-based high-resolution yield estimation: A test of multiple scalable approaches. AGR FOREST METEOROL 2017;247:207-220

31. Tucker CJ. Red and Photographic Infrared linear Combinations for Monitoring Vegetation. REMOTE SENS ENVIRON 1979;8:127-150

32. Gitelson AA. Remote estimation of canopy chlorophyll content in crops. GEOPHYS RES LETT $2005 ; 32$

33. Rouse Jr. JW, Haas R, Schell J, Deering D. Monitoring Vegetation Systems in the Grreat Plains with ERTS. 1974

34. Gitelson AA, Gritz Y, Merzlyak MN. Relationships between leaf chlorophyll content and spectral reflectance and algorithms for non-destructive chlorophyll assessment in higher plant leaves. J PLANT PHYSIOL 2003;160:271-282

35. Gitelson AA, Merzlyak MN. Remote estimation of chlorophyll content in higher plant leaves. INT J REMOTE SENS 1997;18:2691-2697 Vehicle Imagery Reveal Senescence Rate in Bread Wheat. REMOTE SENS-BASEL 2018;10:809 37. Raper TB, Varco JJ. Canopy-scale wavelength and vegetative index sensitivities to cotton growth parameters and nitrogen status. PRECIS AGRIC 2015;16:62-76 
610 Chlorophyll Concentration from Leaf and Canopy Reflectance. REMOTE SENS ENVIRON $6112000 ; 74: 229-239$

612 40. Haboudane D, Miller JR, Tremblay N, Zarco-Tejada PJ, Dextraze L. Integrated narrow-band 613 vegetation indices for prediction of crop chlorophyll content for application to precision agriculture.

614 REMOTE SENS ENVIRON 2002;81:416-426

615 41. Gitelson AA. Wide Dynamic Range Vegetation Index for remote quantification of biophysical 616 characteristics of vegetation. J PLANT PHYSIOL 2004;161:165-173

617 42. Elsayed S, Rischbeck P, Schmidhalter U. Comparing the performance of active and passive 618 reflectance sensors to assess the normalized relative canopy temperature and grain yield of 619 drought-stressed barley cultivars. FIELD CROP RES 2015;177:148-160

620 43. Bending J, Bolten A, Bsreth G. UAV-based Imaging for Multi-Temporal, very high resolution 621 crop surface models to monitor crop growth variability. PHOTOGRAMM FERNERKUN $622 \quad 2013: 551-562$

623 44. Zhao K, Suarez JC, Garcia M, et al. Utility of multitemporal lidar for forest and carbon 624 monitoring: Tree growth, biomass dynamics, and carbon flux. REMOTE SENS ENVIRON $625 \quad 2018 ; 204: 883-897$

626 45. Elsayed S, Elhoweity M, Ibrahim HH, et al. Thermal imaging and passive reflectance sensing to 627 estimate the water status and grain yield of wheat under different irrigation regimes. AGR WATER 628 MANAGE 2017;189:98-110

629 46. Breiman L. Random Forests. MACH LEARN 2001;45:5-32

630 47. Belgiu M, Drăguţ L. Random forest in remote sensing: A review of applications and future 631 directions. ISPRS J PHOTOGRAMM 2016;114:24-31

632 48. Che J, Wang J. Short-term load forecasting using a kernel-based support vector regression 633 combination model. APPL ENERG 2014;132:602-609

634 49. Jiang H, Rusuli Y, Amuti T, He Q. Quantitative assessment of soil salinity using multi-source 635 remote sensing data based on the support vector machine and artificial neural network. INT J 636 REMOTE SENS 2019;40:284-306

637 50. Abrougui K, Gabsi K, Mercatoris B, et al. Prediction of organic potato yield using tillage systems 638 and soil properties by artificial neural network (ANN) and multiple linear regressions (MLR). Soil and 639 Tillage Research 2019;190:202-208

640 51. Guo Y, Yin G, Sun H, et al. Scaling Effects on Chlorophyll Content Estimations with RGB 641 Camera Mounted on a UAV Platform Using Machine-Learning Methods. SENSORS-BASEL $642 \quad 2020 ; 20: 5130$

643 52. Stanton C, Starek MJ, Elliott N, et al. Unmanned aircraft system-derived crop height and 644 normalized difference vegetation index metrics for sorghum yield and aphid stress assessment. J APPL 645 REMOTE SENS 2017;11:26035

646 53. Bendig J, Yu K, Aasen H, et al. Combining UAV-based plant height from crop surface models, 647 visible, and near infrared vegetation indices for biomass monitoring in barley. INT J APPL EARTH 648 OBS 2015;39:79-87 
54. Geipel J, Link J, Claupein W. Combined Spectral and Spatial Modeling of Corn Yield Based on Aerial Images and Crop Surface Models Acquired with an Unmanned Aircraft System. REMOTE SENS-BASEL 2014;6:10335-10355

55. Maimaitijiang M, Ghulam A, Sidike P, et al. Unmanned Aerial System (UAS)-based phenotyping of soybean using multi-sensor data fusion and extreme learning machine. ISPRS J PHOTOGRAMM 2017; 134:43-58

56. Hansen PM, Schjoerring JK. Reflectance measurement of canopy biomass and nitrogen status in wheat crops using normalized difference vegetation indices and partial least squares regression. REMOTE SENS ENVIRON 2003;86:542-553

57. de Jong SM, Addink EA, Doelman JC. Detecting leaf-water content in Mediterranean trees using high-resolution spectrometry. INT J APPL EARTH OBS 2014;27:128-136

58. Elarab M, Ticlavilca AM, Torres-Rua AF, Maslova I, McKee M. Estimating chlorophyll with thermal and broadband multispectral high resolution imagery from an unmanned aerial system using relevance vector machines for precision agriculture. INT J APPL EARTH OBS 2015;43:32-42

59. Neinavaz E, Skidmore AK, Darvishzadeh R, Groen TA. Retrieval of leaf area index in different plant species using thermal hyperspectral data. ISPRS J PHOTOGRAMM 2016;119:390-401

60. Abu-Hamdeh NH. Thermal Properties of Soils as affected by Density and Water Content. BIOSYST ENG 2003;86:97-102

61. Aubrecht DM, Helliker BR, Goulden ML, et al. Continuous, long-term, high-frequency thermal imaging of vegetation: Uncertainties and recommended best practices. AGR FOREST METEOROL 2016;228-229:315-326

62. Kamilaris A, Prenafeta-Boldú FX. Deep learning in agriculture: A survey. COMPUT ELECTRON AGR 2018;147:70-90

63. Holloway J, Mengersen K. Statistical Machine Learning Methods and Remote Sensing for Sustainable Development Goals: A Review. REMOTE SENS-BASEL 2018;10:1365

64. Zha H, Miao Y, Wang T, et al. Improving Unmanned Aerial Vehicle Remote Sensing-Based Rice Nitrogen Nutrition Index Prediction with Machine Learning. Remote sensing (Basel, Switzerland) 2020;12:215 